\title{
Características del liderazgo de la jefatura departamental en matemática de un centro de alto desempeño en educación secundaria en Chile
}

\author{
Leadership's characteristics of the departmental mathematics head \\ of a high-performance center in secondary education in Chile
}

\section{Características da liderança do chefe do departamento de matemática de um centro de alto desempenho no ensino médio no Chile}

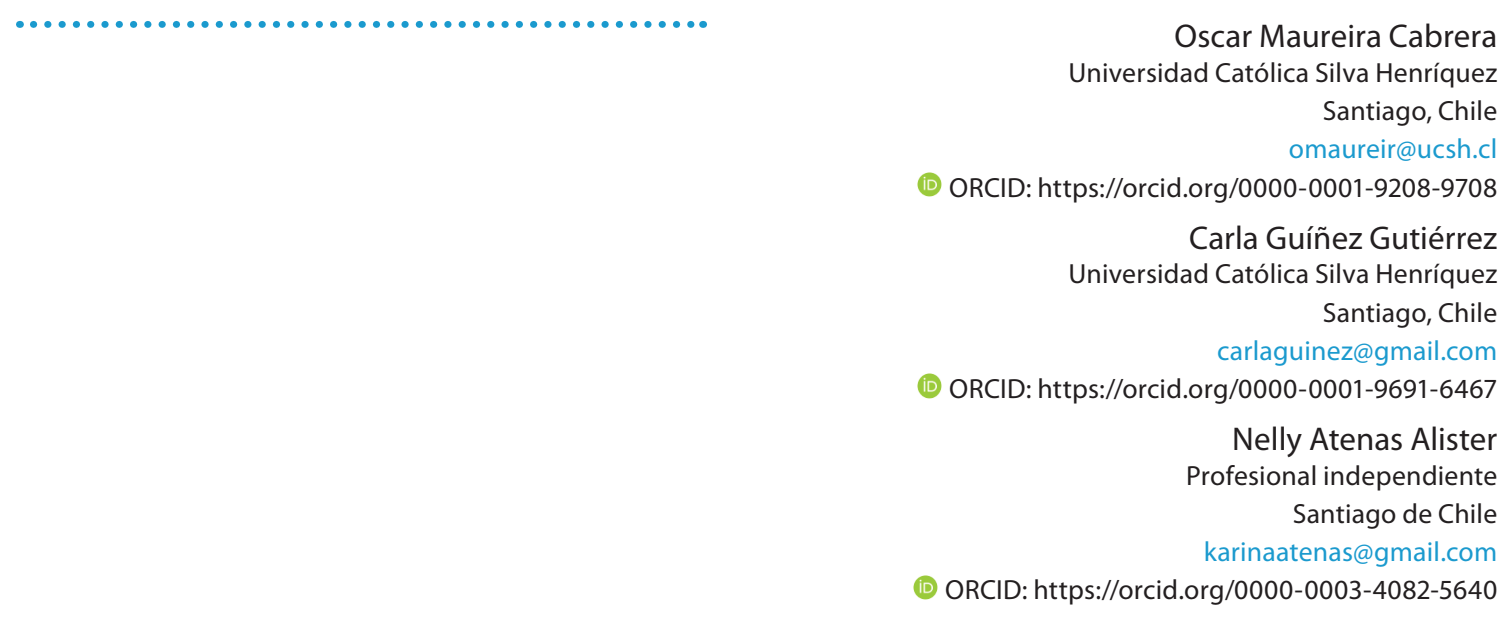

Recibido - Received - Recebido: 15 / 09 / 2021 Corregido - Revised - Revisado: 29 / 10 / 2021 Aceptado - Accepted - Aprovado: 12 / 11 / 2021

DOl: https://doi.org/10.22458/ie.v24i36.3895

URL: https://revistas.uned.ac.cr/index.php/innovaciones/article/view/3895

\begin{abstract}
Resumen: El objetivo del siguiente trabajo, es caracterizar el liderazgo y factores contextuales del jefe del Departamento de Matemática de un centro de educación secundaria chileno, con trayectoria de desempeño escolar notable, que ha institucionalizado la departamentalización como estrategia de sostenibilidad de mejora. Mediante un enfoque descriptivo, basado en un estudio de caso único, se realizaron entrevistas semiestructuradas a la jefa de departamento y encargadas de la Unidad Técnico-Pedagógica del centro. Los resultados indican que tal cargo y unidad departamental responde, de manera simultánea, a lineamientos políticos internos de la dirección escolar y a características de liderazgo docente que fortalecen la colaboración entre pares. Como principal conclusión, se reconoce la potencialidad didáctica del quehacer departamental y el valor de las competencias de liderazgo pedagógico, asumido por la jefatura para sustentar la efectividad de la enseñanza de matemática y favorecer el desarrollo profesional docente situado.
\end{abstract}

Palabras clave: Liderazgo, calidad de la educación, gestión educacional, relaciones entre pares, escuela secundaria. 


\begin{abstract}
This works' objective is to characterize the leadership and contextual factors of the head of the Department of Mathematics of a Chilean secondary education center, with a record of notable school performance, who institutionalized departmentalization as a sustainability improvement strategy. Using a descriptive approach, based on a single case study, we conducted semi-structured interviews with the head of the department and those in charge of the Technical-Pedagogical Unit of the center. The results indicate that such position and departmental unit respond simultaneously to the school leadership's internal political guidelines and to characteristics of teacher leadership that strengthen the collaboration among peers. As the main conclusion, the didactic potential of the departmental task and the value of the pedagogical leadership competencies, assumed by the leader to sustain the effectiveness of mathematics teaching and favor the teacher's professional development, is recognized.
\end{abstract}

Keywords: Leadership, quality of education, educational management, peer relations, secondary school.

Resumo: O objetivo do trabalho a seguir é caracterizar a liderança e os fatores contextuais do chefe do Departamento de Matemática de um centro de ensino médio chileno, com um histórico de desempenho escolar notável, que institucionalizou a departamentalização como estratégia de melhoria da sustentabilidade. Por meio de uma abordagem descritiva, e a partir de um estudo de caso único, realizamos entrevistas semiestruturadas com o chefe do departamento e os responsáveis pela Unidade Técnico-Pedagógica do centro. Os resultados apontam que tal função e unidade departamental respondem, conjuntamente, às diretrizes políticas internas da liderança escolar e às características da liderança docente que fortalecem a colaboração entre pares. Como conclusão principal, reconhecemos o potencial didático da tarefa departamental e o valor das competências da liderança pedagógica, assumidas pela liderança para sustentar a eficácia do ensino da matemática e propiciar o desenvolvimento profissional do professor.

Palavras-chave: Liderança, qualidade da educação, gerenciamento educacional, relações entre pares, ensino médio.

\title{
INTRODUCCIÓN
}

El estudio del liderazgo escolar se ha extendido, en el caso de la educación secundaria, a otros potenciales agentes de cambio más allá de las funciones de las personas directores, tales como jefes de unidades departamentales, coordinadores de proyectos o de ciclos de etapas de enseñanzas, personas docentes tutores y de aula (Anderson, 2012; Crawford, 2012). Esta tendencia se manifiesta, por un lado, en la búsqueda de líderes alternativos que colaboran con la dirección de los centros (Grootenboer, EdwardsGroves y Rönnerman, 2015) y, por otro, en el aumento de cargos medios, en algunos países, para apoyar a tal dirección (Mifsud, 2017; Lahtero, Lång y Alava, 2017).

Sin embargo, en Iberoamérica y, particularmente en Chile, es escasa la investigación del liderazgo en otros roles que interactúan entre dirección y docentes, en la estructura organizativa de los centros, además de su potencial contribución a procesos de mejora escolar, como lo es el liderazgo medio (Cortez y Zoro, 2016) y/o de la persona docente (Harris, Ng, Jones y Thanh, 2017). Por consiguiente, no existen políticas que lo promuevan y definan. Prueba de ello, es que los cuerpos legales solo reconocen la función directiva superior, unidad técnico-pedagógica de los centros y la función docente propiamente tal, aunque se han identificado variedad de roles y funciones, como mandos medios, que podrían ser un sustantivo aporte a la mejora (Cancino y Vera, 2017).

En el caso de la estructura organizacional de los centros escolares de educación secundaria, dada su especificidad curricular, se ha concretado tradicionalmente en departamentos. Así, dos son los factores que pueden potenciar su desarrollo y contribuir a la mejora escolar. Por una parte, las capacidades de gestión y liderazgo directivo, que focalizan sus esfuerzos en crear condiciones institucionales para el desarrollo de una cultura colaborativa (Krichesky y Murillo, 2018) y, por otro, la capacidad de las jefaturas departamentales para influir en desarrollo profesional de pares, mediante comunidades de aprendizaje (Hairon, Goh y Chua, 2015).

En este sentido, uno de los actores fundamentales en la explicación de la mejora de la eficacia escolar, es el profesorado. Su desempeño en el aula, es determinante para la calidad del aprendizaje, pero este 
desempeño se asocia a múltiples variables. Una de ellas estaría fuera del aula y se enmarcaría en una de las perspectivas del liderazgo docente, que según Cooper et al. (2016), se relaciona con la capacidad de influir en las prácticas pedagógicas de toda la escuela.

El liderazgo docente, para Bernal e Ibarrola (2015), es uno de los principales propósitos de la gestión educativa y se vería reflejado en su capacidad para influir en los colegas, directores y otros actores de la comunidad educativa, tanto individual como colectivamente, para impulsar prácticas de enseñanza de mejora escolar (Bolívar, 2019). Wenner y Campbell (2017) indican que los perfiles del profesorado se relacionan e interactúan de manera influyente, dentro y fuera del aula, al ser actores críticos de procesos del aprendizaje docente, que buscan desarrollar prácticas de calidad, cuyas funciones se enmarcan en concepciones de liderazgo medio (Leithwood, 2016).

Ahora bien, aunque son diversas las acepciones del liderazgo docente, aún no existe una definición consolidada. Harris et al. (2017) precisan que la mayoría comparte características comunes como, por ejemplo, que es un proceso de influencia, practicado en colaboración, con mutua confianza y se dirige a mejorar la calidad pedagógica y los aprendizajes escolares.

La práctica de este liderazgo, según York-Barr y Duke (2004), se manifiesta en cargos, roles y canales de comunicación formales e informales, de la cotidianidad del quehacer escolar, destacándose a los jefes departamentales de las asignaturas. Las dimensiones de la práctica, según los autores, se deben concentrar en áreas de gestión, coordinación, currículo, desarrollo profesional docente, gestión del cambio, vínculo con actores de la comunidad educativa, contribuciones a la profesión y formación docente, antes de asumir tal cargo. Respecto a los métodos de influjo, se destacan las formas de apoyo, en la colaboración y desarrollo de relaciones de confianza entre pares. Apoyo que se enmarcaría en lo funcional, formativo y también en lo psicosocial.

En relación con los factores individuales que condicionan la efectividad del liderazgo docente, en sus diferentes ámbitos de acción, Harris et al. (2017) destacan el conocimiento de los contenidos de los docentes, procedimientos, saber cómo y la motivación, como aquellos que afectan positivamente la habilidad de liderar. En cambio, variables como: edad, género, formación académica, experiencia docente, cargo formal y la percepción de liderazgo docente, no son del todo concluyentes, respecto de su efectividad.

La experiencia del liderazgo docente, bajo la perspectiva del liderazgo distribuido, da cuenta de los roles, estrategias y las limitaciones del profesorado como líderes en diversos ámbitos, por ejemplo, al sentirse responsables de fomentar el desarrollo profesional en las escuelas (Supovitz, 2018). En cuanto a estrategias, se orientaron procesos sin dejar de verse como docentes de aula y con foco en el aprendizaje estudiantil. Sobre las limitaciones, se señala que, en ocasiones, por no tener un rol posicional en la estructura, se sintieron incapaces de obtener avances y ciertas tensiones para no afectar la relación con sus pares.

Sobre los factores de efectividad de los departamentos disciplinarios, como unidad organizativa, Harris, Jamieson y Russ (1995) hallaron la construcción de una visión para el departamento, el monitoreo del desempeño de los integrantes, mediante la observación de sus prácticas en el aula, el uso de los resultados de observación para un diálogo sobre dichas prácticas y, la mantención del registro del desempeño individual del estudiantado, lo que permite la verificación del rendimiento a través del tiempo. En tanto Sammons, Hillman y Mortimore (1995) detectaron que las jefaturas eficaces poseían altos niveles de habilidades de trabajo en equipo, relaciones interpersonales, altos niveles de confianza y criterios para evaluar iniciativas externas, que pudiesen producir sobrecarga de trabajo.

Las características que identifican y realizan las jefaturas departamentales, para Vanblaere y Devos (2016), se manifiestan en las influencias que favorecen la colaboración docente y la creación de ambientes de colegialidad. Flückiger, Lovett, Dempster y Brown (2015), por su parte, precisan que una acción clave de las jefaturas, se sitúa en dar una dirección estratégica y desarrollo profesional docente a pares 
para la enseñanza de la disciplina, optimizar el uso de diferentes recursos y constituirse en líderes del aprendizaje para otros.

En el caso chileno, cabe precisar que los departamentos de asignaturas, a raíz de la tendencia de enmarcar ciertas competencias clave de roles directivos para asegurar la gestión de calidad en los centros, el Área de Educación de la Fundación Chile (2006) precisó que dicho cargo debe estar ligado a la Unidad Técnico-Pedagógica (UTP) y su responsabilidad mayor, se centra en implementar y cumplir el proyecto curricular del área. Además, siguiendo a Araya (2015), esta responsabilidad se estructuraría por medio de los siguientes ámbitos de acción y competencias:

TABLA 1

Competencias funcionales y conductuales del jefe(a) de departamento

\begin{tabular}{l|l}
\multicolumn{1}{c}{ Funcionales } & \multicolumn{1}{c}{ Conductuales } \\
\hline Ámbitos de Gestión & \\
\hline Asegurar la existencia de información útil para la toma oportuna de decisiones & Compromiso ético social \\
\hline Gestionar el personal & Orientación a la calidad \\
\hline Planificar y coordinar las actividades de su área & Autoaprendizaje y desarrollo profesional \\
\hline Administrar los recursos de su área en función del PEl & Liderazgo \\
\hline Asegurar la disponibilidad de recursos pedagógicos a los docentes & Responsabilidad \\
\hline Coordinar aspectos administrativos y operativos & Adaptación al cambio \\
\hline Supervisar la implementación de los programas en el aula & Asertividad \\
\hline Asegurar la calidad de las estrategias didácticas en el aula & Iniciativa e innovación \\
\hline Elaborar y gestionar proyectos de mejoramiento & \\
\hline Ámbito Curricular & \\
\hline Asegurar la implementación y adecuación de planes y programas & \\
\hline Adecuar estrategias de enseñanza para el aprendizaje & \\
\hline Mejorar las estrategias de acuerdo con los resultados & \\
\hline Gestionar proyectos de innovación pedagógica & \\
\hline Fuente: Rediseño del rol y función del jefe de departamento de asignatura. Estudio de caso (Araya, 2015. p. 41).
\end{tabular}

Araya (2015) evidenció, que la función de los jefes de departamentos no está delimitada desde el campo de acción del rol y son inexistentes, los criterios manifiestos de los departamentos que establecen el uso de diferentes recursos, métodos y tecnología para la enseñanza. Asimismo, no existen mecanismos institucionalizados (protocolos de acción) para el desarrollo profesional del profesorado, aspectos que configuran una tendencia más a la gestión administrativa y con escasa manifestación del quehacer departamental, como comunidad profesional de aprendizaje.

Bielecka (2015), por su parte, al indagar las características del liderazgo intermedio, focalizándose en el rol de coordinación de la unidad departamental halló, a nivel escolar, que existía una cultura de altas expectativas, gran compromiso de coordinadores, profesorado y familias hacia la mejora del aprendizaje estudiantil. Sobre la coordinación y el departamento, se precisa, que entre sus integrantes y la coordinadora existe autonomía para el trabajo del profesorado, desarrollo de actividades pedagógicas creativas y asimilación de los objetivos planteados por el equipo, ajustándose a los cambios en pos de la mejora del programa disciplinar. Como prácticas en el rol de coordinación, se obtuvo que existe seguimiento del desempeño de docentes en aula, incluyéndose actividades de observación, realimentación 
y diálogo constructivo. Además, se favorecía y modelaba el trabajo de equipo y un estilo relacional más horizontal que vertical.

Chávez, Faúndez, Martínez y Zett (2018), en tanto, concluyeron que la estructura departamental, como medio para el desarrollo profesional docente, corresponde a una estrategia para mejorar los resultados de aprendizaje, cuyos principios de funcionamiento se enmarcan en comunidades profesionales de aprendizaje. Aunque el desarrollo profesional docente respondía más bien a aspectos contingentes, sin alcanzar una meta de aprendizaje colectivo. Limitaciones explicadas, por factores institucionales asociados a recursos (tiempo) y articulación (coordinación) entre el nivel institucional y sectorial, respecto a la mejora escolar.

Bajo el marco del liderazgo distribuido, Barrios, Escare, Espinoza y Jiménez (2018) identificaron beneficios y dificultades del trabajo de los jefes de departamentos, señalando que la toma de decisiones de los jefes de departamentos, es incipiente y sectorial como manifestación del liderazgo distribuido, pues sigue prevaleciendo la perspectiva institucional como recurso de decisión final. Las exigencias de resultados en pruebas estandarizadas externas son, por lo general, atribuibles al rol de los jefes de departamentos, lo que genera tensiones y oportunidades en el desempeño. Sobre el quehacer de los departamentos, este permanece en nivel de cooperación y gran parte de las tareas en la unidad, son asumidas voluntaristamente por el jefe(a).

Considerando, entonces, los diferentes antecedentes referidos, tanto a vacíos de conocimiento como a las potencialidades del rol de las jefaturas de los departamentos, que respalda la literatura, principalmente anglosajona, sobre el liderazgo docente y/o medio en el contexto de la educación secundaria; el siguiente trabajo, que es parte de un proyecto más amplio, tiene como propósito caracterizar el rol, función y liderazgo del jefe del departamento de matemática en una institución de educación secundaria chilena municipal (pública) ubicada en el norponiente de Santiago, con una trayectoria de desempeño escolar notable durante una década. Los objetivos específicos, se enmarcan en identificar el origen y funciones de la jefatura, así como describir el liderazgo y prácticas de esta.

\section{MATERIALES Y MÉTODOS}

La investigación se sustenta en una metodología cualitativa, que utiliza un enfoque descriptivo, pues se pretende analizar la forma en que se manifiesta el fenómeno sobre el cual se indaga, para desarrollar descripciones detalladas y precisas sobre situaciones, interacciones y/o conductas observadas (Hernández, Fernández y Baptista, 2010), a partir de lo que indican los participantes, en cuanto a las características asociadas al rol, función y liderazgo del jefe del departamento, de una institución de educación secundaria chilena.

Así, se empleó un método de caso único, para dar cuenta y analizar de modo intencionado un fenómeno específico, llevando a cabo un examen sistemático y profundo de casos asociados a un aspecto particular de estudio (Hernández y Mendoza, 2018). En el contexto de esta investigación, corresponde a una figura de liderazgo -el jefe de departamento- al interior de una entidad educativa particular.

La muestra empleada del estudio, es intencional y corresponde al departamento de matemática de un establecimiento educacional, ubicado en el sector norponiente de Santiago, el cual depende de una corporación municipal. Esta institución oferta a la comunidad, una modalidad de enseñanza polivalente, con formación científico-humanista (HC) y técnico profesional (TP), atendiendo aproximadamente a 850 personas, cuyas edades fluctúan entre los 12 y 17 años. Además, se encuentran distribuidas, entre los cursos de $7^{\circ}$ grado de educación primaria al último grado de educación secundaria. 
Dentro de los criterios para la elección del establecimiento y su departamento de matemática, es posible indicar que ellos:

a) Se caracterizan por poseer un alto Índice de Desarrollo Educativo (Valenzuela, Bellei y Allende, 2016). De acuerdo con la Agencia de Calidad de la Educación (2018), el establecimiento está categorizado en el nivel de desempeño alto;

b) Atienden a un estudiantado con un elevado Índice de Vulnerabilidad Escolar (IVE) (JUNAEB, 2018). EI IVE de este establecimiento, en el año 2018, alcanzó el 78,86 \% en enseñanza básica y el 81,85 \% en educación media (JUNAEB Abierta, s/f) y,

c) Evidencian un desempeño en matemática, superior a establecimientos similares. Según datos de la Agencia de Calidad de la Educación (2018), este establecimiento posee un estudiantado con resultados sobresalientes a lo esperado, siendo superiores a instituciones, con atributos similares en las evaluaciones estandarizadas de matemáticas aplicadas en Chile, por casi una década.

Los informantes seleccionados para este caso de estudio, corresponden a la jefa del Departamento de Matemática (líder departamental) y las encargadas de la Unidad Técnico-Pedagógica (líderes UTP 1 y 2).

Por otro lado, el trabajo de campo se desarrolló en un trimestre académico, en el que se aplicaron instrumentos de producción de información, construidos desde el equipo de investigadores del proyecto mayor al que adscribe este caso particular, los cuales fueron inicialmente, sometidos a validación de expertos y, posteriormente, al pilotaje del tipo marcha blanca, con directivos y un profesorado perteneciente a un establecimiento que cumplía con los criterios antes indicados y que no era parte de la investigación central.

La técnica de producción de información empleada, corresponde a la entrevista semiestructurada, aplicada a jefes de departamento y encargados de UTP. En lo que refiere a la entrevista, esta puede ser entendida como una comunicación interpersonal, establecida entre el investigador y el sujeto de estudio para obtener respuestas verbales a las interrogantes planteadas sobre el problema propuesto (Canales, 2006), de modo que la misma, constituye un instrumento técnico que adopta la forma de un diálogo coloquial, pero cuyo propósito consiste en un fin determinado, con preguntas elaboradas, teniendo en cuenta la pregunta de investigación y los objetivos a alcanzar.

Los focos centrales de los instrumentos aplicados, se detallan en la Figura 1, asociados a los objetivos de investigación, por lo que las categorías dan respuesta a estos:

Figura 1. Objetivo y categorías de análisis

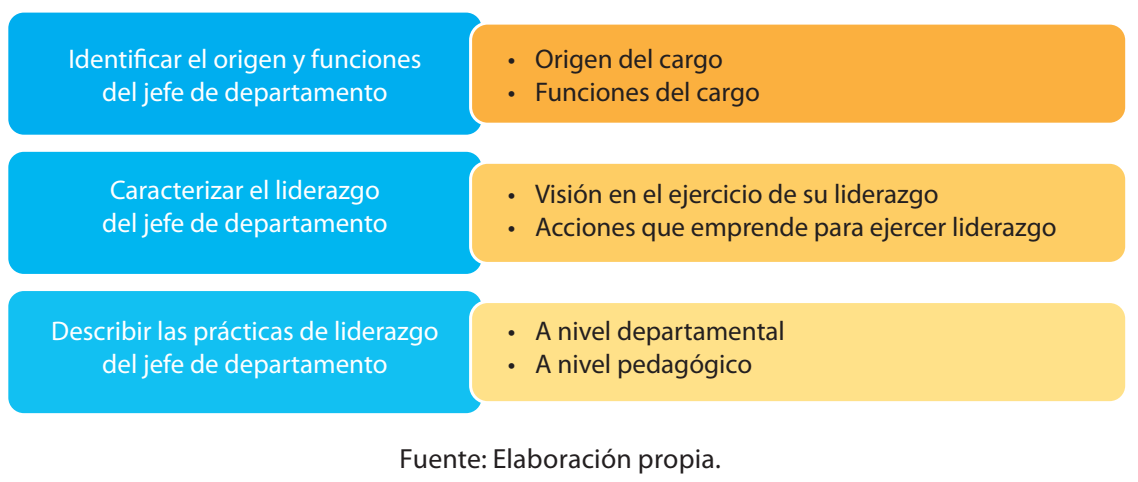


En virtud de lo que se indagó en esta investigación, se empleó el análisis de contenido para interpretar la información, que emergió de las conversaciones entabladas con las participantes del estudio, identificándose los sentidos manifiestos o implícitos, cuyos contenidos inferenciales son expresados con patrones de significados, "los cuales pueden ser interpretados como indicadores o contextos" (Díaz, 2018, p. 126). Esto favorece la descripción del fenómeno estudiado en un determinado contexto (García, Alvira, Alonso y Escobar, 2015). Todo lo anterior respaldado con el software Atlas.ti 7.

\section{DISCUSIÓN DE RESULTADOS}

El principal hallazgo de este trabajo evidenció, que la jefatura departamental, más allá de ciertas precariedades institucionales en el origen de la formalización de la departamentalización, desplegó una serie de prácticas propias del liderazgo docente, que se conjugaron con estrategias del liderazgo directivo (director) para la sostenibilidad de la mejora escolar.

\section{Origen y funciones del jefe de departamento de matemática}

Los datos obtenidos llevaron a establecer, que el origen del cargo, en el contexto estudiado, se encontraba en un estadio incipiente, pues fue fruto de una política interna que buscaba configurar una estructura formalizada del cargo y su labor al interior del establecimiento, para lo cual se destinaron jornadas de trabajo protegidas, que reunían a los jefes departamentales con el equipo directivo, conformando una instancia de coordinación interdepartamental. De manera paralela, se generó una asignación de tiempo semanal para el trabajo interno como departamento, que respondía a las necesidades de generar espacios protegidos para la promoción de comunidades profesionales y disciplinares: "el departamento en el fondo está funcionando recién este año... Como departamento en sí, con estas reuniones, porque antes nunca teníamos la oportunidad de reunirnos para hablar de nuestro quehacer en cuanto a matemáticas o poder cambiar experiencias". (Líder departamental).

En el establecimiento estudiado, el cargo en sí no implicaba una remuneración económica para el jefe de departamento. A esto, se sumó la ausencia de un perfil formal que regulara y resguardara la labor a desarrollar, pues fue el equipo directivo el que seleccionó y nombró a la persona encargada de desempeñar el cargo, a partir de ciertas cualidades, como contar con el reconocimiento y validación de sus pares, o evidenciar buenos resultados en las pruebas estandarizadas a las que se sometía el establecimiento: "se ha intencionado que el jefe de departamento tenga un buen discurso, se alinee con el proyecto educativo y se alinee con el Equipo Directivo" (Líder 1 UTP).

Cuando se consultó respecto a los lineamientos que determinaban las labores propias del jefe de departamento, la líder departamental indicó, que su funcionamiento no estaba formalizado en un documento o espacio formal que orientase sobre qué labores realizar y tampoco, existían evaluaciones formales que ayudaran al desempeño de sus funciones: "La verdad es que así como 'te voy a evaluar por tu cargo de jefe' no existe, no... Más allá de decirme 'lo estás haciendo bien como jefa de departamento' eso fue, fue todo. No hay, así como una reunión 'ya, vamos a evaluarlos como jefes de departamento'". (Líder departamental).

Ahora bien, sobre las funciones específicas del jefe de departamento, fue posible observar que para el equipo directivo, fue necesario que el candidato a ocupar este cargo, tuviese la capacidad de liderar el proceso pedagógico curricular de la disciplina, alinear discursos entre docentes, preocuparse de los resultados y espacios evaluativos del estudiantado: "es superimportante [que] en esa reunión con todos 
los profes de matemática, de lenguaje o los diferentes departamentos estén alineados con el discurso de la asignatura" (Líder UTP 2).

Cuando se analizó la opinión de la líder departamental respecto a sus funciones, fue posible apreciar que, para ella, una de sus principales funciones se encontraba asociada a tópicos curriculares que debían cubrir como equipo, principalmente, en lo referido a trabajar los contenidos que requiere el estudiantado para alcanzar las metas académicas propias de un colegio de alto desempeño: "La meta es cumplir con todo lo que nos pidan con respecto a los directivos, y que son hartas cosas que nos van pidiendo, y metas que nosotros mismos [nos proponemos], no sé poh, si ya nos fue bien en el SIMCE un año, cada vez nuestra meta es más alta, y más agotadora también". (Líder departamental).

\section{Características del liderazgo desplegado por la jefa del Departamento de Matemática}

Este aspecto fue dividido en dos ámbitos de análisis: visión respecto al liderazgo ejercido y acciones emprendidas para desplegar su liderazgo. En relación con el primer punto, fue posible observar que esta líder departamental se caracterizó, por tener una visión en el ejercicio de su liderazgo, intencionada y enfocada a la mejora de aprendizajes estudiantiles; para esto, priorizaba en sus colegas, la entrega de aprendizaje de calidad y significativo que perdurasen en el tiempo.

Bueno, las prioridades siempre son los niños, la enseñanza de los niños, entregarles calidad más que cantidad, porque eso siempre se nos ha pedido a nosotros... así es que eso es lo que yo he transmitido también a los jóvenes que vienen recién llegando y eso, es nuestra prioridad, es que el niño aprenda, que aprenda bien, que tenga un aprendizaje significativo, que se sienta bien, que sea protagonista de su aprendizaje, que participe, que esté en un ambiente grato. (Líder departamental).

Respecto al segundo punto, fue posible identificar, que una de sus acciones apuntó al monitoreo y acompañamiento de sus colegas, permitiéndole interactuar fluidamente con ellos. Con el tiempo, se pudieron establecer espacios de observación y realimentación de clases para dialogar, reflexivamente, sobre las prácticas de enseñanza-aprendizaje, implementadas al momento de enseñar y el modelamiento de estas. "En un principio, cuando empezamos con las reuniones los martes, utilizábamos los dos periodos en reunión, entonces igual veíamos como que era improductivo... y después se generó el tema de ir a observar clases... La observación entre pares ha sido una muy buena estrategia [para mejorar las prácticas de los docentes], invitarlos a ver a los más antiguos". (Líder departamental).

Cuando se consultó sobre la interacción entre la líder departamental y los docentes de su equipo, se pudo inferir que ella generaba un clima que favorecía la reflexión compartida, por ejemplo, potenciar el intercambio de experiencias pedagógicas y docentes, promover la reflexión sobre el ser y hacer profesional, incitar la colaboración en la elaboración de ideas y sugerencias para la mejora: “[Buscaba] generar un ambiente agradable, que se sintieran cómodos, que pudieran explayarse, y que si teníamos que reírnos, nos reíamos; si nos teníamos que colocar serios, nos poníamos serios. Es eso, es lograr ese ambiente". (Líder departamental).

También, se pudo apreciar que ella, innatamente, desplegó acciones de conducción participativas, pues favorecía la generación de espacios para el diálogo pedagógico entre pares, respetando las individualidades del profesorado y promoviendo, una cultura de apertura a la crítica, donde los errores son oportunidades de aprendizaje: "Como hay dos profesores que hacen segundos medios distintos, se van tratando de colocar de acuerdo para ir al mismo ritmo, para compartir guías, para compartir pruebas...o 
cómo se está enseñando 'oye, dime tú cómo estai enseñando esto, porque a mí no me está funcionando'". (Líder departamental).

También fue posible indicar, que la líder departamental impulsaba acciones para levantar propuestas en equipo, con la finalidad de mejorar el funcionamiento interno del establecimiento, como garantizar espacios y tiempo para trabajar en las labores propias del departamento: "se dieron los cambios porque nosotros solicitábamos poder reunirnos con nuestro departamento, porque como le digo era siempre conversación de pasillo, nada formal, algo muy muy a la rápida". (Líder departamental).

Otra acción estuvo dirigida a resguardar espacios propicios para efectuar la realimentación entre pares, luego de realizadas las observaciones de la práctica pedagógica de las persona docentes adscritas al departamento. "Nos faltaba el horario para retroalimentar, entonces hablamos para conseguir que nos den el espacio para poder retroalimentar a los profesores, porque no sacamos nada con ir a observarlos y no poder juntarnos tranquilos a conversar un rato de cómo fue la observación, o qué le puede uno ayudar o aportar". (Líder departamental).

Cabe destacar, que una nueva acción de la líder departamental, estuvo relacionada con la capacidad para desempeñarse como nexo entre el profesorado y el equipo directivo, ayudando a transmitir indicaciones, solicitudes y requerimientos al profesorado, respecto a lo que se espera de ellos, para lo cual empleó diversas estrategias de difusión: "Aparte de reuniones con los jefes de UTP y directivos los días martes, es también transmitir esa información a los profesores, ir recordándoles cosas que hay que hacer, yo generalmente mando correos, les voy avisando a todos en general, hay que hacer esto, que las pruebas de nivel, entonces también soy como una ayuda de memoria". (Líder departamental).

Además de prácticas para influir en el profesorado del departamento, en lo referente a las prácticas desplegadas por la jefa del Departamento de Matemática, es posible señalar que se logró identificar la existencia de:

a) Prácticas que apuntan a lo pedagógico entre los diversos miembros del equipo, con el propósito de resguardar el trabajo de aula y la relación del estudiantado.

Se evidenció que la líder departamental, se preocupaba por concentrar la labor de los docentes que dirige, en aspectos propios del proceso de enseñanza-aprendizaje, entendiendo la responsabilidad socio-profesional que poseen como formadores de sujetos integrales, por lo que invitaba a la reflexión y cuestionamiento constante entre sus pares. "Ahora como una de las últimas reuniones recuerdo que era, que como somos muy academicistas, tenemos que trabajar más el tema de los valores, y hacer que el alumno, ehhhh reforzarle los valores que les dan en la casa y que lo sienta así, y el que no lo tiene, que no lo trae de la casa, poder entregárselo nosotros". (Líder departamental).

b. Prácticas instrumentales/departamentales que tienen por finalidad sentar bases para el funcionamiento y las relaciones entre los miembros su equipo.

Acá se pudo identificar, en primer lugar, una líder departamental que a partir de las atribuciones provistas por su cargo, intentó resguardar espacios para la confianza y cuidado de sus colegas: "Les pregunto cómo están, tuviste algún problema, ehhhh. Una conversación más relajada pero también de pasillo. De repente, yo veo a mi profesor angustiado o aproblemado. ¿Qué pasó?, ¿cuál es el problema? Solucionémoslo, ya. A veces no puedo tomar decisiones, porque no soy jefe de UTP, ni soy directivo, pero sí le puedo guiar". (Líder departamental).

En segundo lugar, desplegó habilidades de coaching o mentoría, principalmente al profesorado novel que integraba su equipo, para fomentar la mejora de la enseñanza de matemática y el desarrollo profesional de sus pares: "tengo un profesor que recién está, es nuevito, y le fui a ver su primera clase... lo invité a ver una de las mías (...), yo después le fui a ver otra clase y mejoró, pero significativamente". (Líder departamental). 
Para finalizar y en tercer lugar, se pudo observar que la líder departamental demostró cualidades de liderazgo que practicó, cotidianamente, con el profesorado: promoviendo la escucha y el diálogo efectivo, practicando la empatía con sus colegas, generando espacios de trabajo colaborativos, cuestionando y reflexionando asiduamente, las implicancias de su cargo y quehacer como referente que encabeza un equipo de personas docentes, presionados en mantener excelentes resultados académicos, tanto en pruebas internas como estandarizadas.

A partir de los resultados descritos, fue posible indicar que, en relación con el origen y las funciones asociadas a la líder departamental estudiada, dichos aspectos estaban definidos discrecionalmente, por el director y equipo directivo, aunque la inquietud de formalizar tal unidad era agenciada por la líder departamental, en su rol inicial de docente. Característica que, según Wenner y Campbell (2017), corresponde a un atributo de los perfiles docentes que, como manifestación de su liderazgo, interactúan de modo influyente dentro y fuera del aula. Se constituyen, entonces, en actores críticos que buscan desarrollar prácticas docentes de calidad (Leithwood, 2016).

La literatura señala que, quienes asumen las jefaturas, deben poseer habilidades para guiar e influir en sus colegas, aceptando el reto que implica esta función (Cortez y Zoro, 2016; Sepúlveda y Volante, 2019). Por tanto, este caso evidenció lo señalado por Cancino y Vera (2017), sobre la estructura políticoadministrativa de muchos centros escolares chilenos, al no visibilizar formalmente, la labor de los jefes o coordinadores a cargo de los departamentos disciplinares.

En el contexto estudiado, dicho cargo se movía en el borde de la informalidad. La líder tenía certeza sobre el nivel de reconocimiento simbólico en su comunidad escolar, pero de forma paralela, experimentaba la ausencia de reconocimiento a nivel contractual, que compensase la responsabilidad de la labor. Tampoco poseía condiciones laborales propicias a la nueva tarea, a la fecha no disponía de un horario protegido para concentrarse en las tareas asociadas a nuevas responsabilidades. Esto refleja, que las políticas educativas y normativas desconocen la complejidad del rol vinculado a este tipo de dirección, quedando al arbitrio de los directivos, el tipo de escenario en que se desenvuelven al asumir como jefes. La mayoría de sus características funcionales se contradicen con lo que indican TichnorWagner, Harrison y Cohen-Vogel (2016), pues la dirección debe asegurar espacios, horarios y claridad de propósitos para potenciar prácticas departamentales efectivas.

Al reportar las características del liderazgo, desplegadas por la líder departamental, la evidencia obtenida permitió afirmar, que ella poseía características propias de los líderes de departamentos eficaces de las que refiere Bielecka (2015), así como las que hace unas tres décadas encontraron Harris et al. (1995) y Sammons et al. (1995), pues trabajaba en crear una visión compartida con sus colegas y para su departamento, se preocupaba por monitorear y acompañar el desempeño de su equipo, a través de la observación de las prácticas en aula y el resultado de la observación, lo usaba para promover un diálogo reflexivo que potenciara la mejora de las prácticas del departamento, asumiendo espacios y tiempos para compartir la realimentación al trabajo hecho por sus pares.

En cuanto a las prácticas que implementaba la líder analizada, para lograr influir al interior de su equipo, se comprobó que ella favorecía la colaboración para generar climas de confianza, participación y compromiso entre sus colegas, mediante la exploración del sentir de los docentes en las reuniones de equipo. Acciones que, según Harris et al. (2017), se enmarcarían en la categoría de apoyo, con dominio de lo psicosocial. En lo concerniente al apoyo formativo para el desarrollo profesional docente, esta líder logró gestionar acciones de asesoramiento de las prácticas docentes, mediante el acompañamiento y modelaje permanente con sus docentes. 


\section{SÍNTESIS Y REFLEXIONES FINALES}

En relación con el objetivo de identificar el origen y funciones de la jefatura departamental, esta se origina por un lineamiento político interno, que busca formalizar la departamentalización dentro de la estructura organizativa del centro, al desarrollarse de manera implícita. En este sentido, la jefatura se configura, como un enlace entre la dirección escolar y el profesorado de matemática. Tal rol se nutre en su desempeño, por la coordinación interdepartamental de carácter institucional y las jefaturas UTP.

Si bien no existe un documento formal que explicite el perfil y funciones de la jefatura, a juzgar por los resultados, es posible afirmar que una de las principales funciones, sería la de coordinar el quehacer curricular y docente del departamento para mantener o subir los aprendizajes medidos, tanto por pruebas internas como externas estandarizadas. Luego, la de realimentar la práctica pedagógica de sus pares, con un fin formativo.

Respecto del objetivo, sobre describir características de liderazgo que despliega la jefatura en su desempeño, se observan atributos propios del liderazgo docente. Tal jefatura cuenta con el reconocimiento de la dirección escolar del centro y también de sus pares, por su alta calidad docente, lo que le confiere legitimidad y aceptación al interior de la comunidad educativa. Asimismo, su cercanía y estilo comunicacional, le permite establecer y desarrollar lazos de confianza relacional, favoreciendo la creación de ambientes propicios, para implicar a sus pares en la mejora de las prácticas pedagógicas, en el aprendizaje de matemática. Además, posee la capacidad de acompañar y guiar al profesorado novel.

Finalmente, los departamentos de asignatura podrían llegar a cumplir un rol estratégico, en el logro de los objetivos institucionales de mejora de los aprendizajes escolares, pero su desarrollo está mediado por factores de políticas, tanto externos (por ejemplo, normativa sobre responsabilidades para cargos intermedios) como internos del centro (por ejemplo, perfil de cargo intermedio, monitoreo y evaluación de dicha labor, desarrollo formativo para el cargo y sus funciones). No obstante, un factor clave en la efectividad de la unidad, como se infiere de este caso, se focaliza en las competencias de quien la dirija, sobre todo en cómo se legitima ante sus pares a través de saberes, como la enseñanza de la disciplina in situ (conocimiento pedagógico del contenido) y de los resultados de aprendizaje evidenciados que logra el estudiantado.

Si bien el estudio se concentró en describir las características del liderazgo de un rol de jefatura departamental en un centro de educación secundaria de alto desempeño escolar, son múltiples y diversas las líneas de investigación que surgen para el estudio del liderazgo, bajo la perspectiva del liderazgo docente o medio, tanto en puestos de aula de centros escolares como intermedios. Sobre todo, asociados a los posibles efectos en los procesos de enseñanza-aprendizaje. Además de analizar si existen diferencias sustantivas en sus características, según nivel escolar o área del conocimiento en que se enmarcan.

\section{AGRADECIMIENTO}

Se agradece el financiamiento aportado por FONDECYT (Proyecto N 1180687): La contribución del liderazgo pedagógico de los jefes de departamentos disciplinarios en los procesos de mejora escolar en educación secundaria.

\section{REFERENCIAS}

Agencia de la Calidad de la Educación (2018). Agencia de la Calidad de la Educación da a conocer categorías de desempeño 2018. Recuperado de https://www.agenciaeducacion.cl/noticias/ agencia-calidad-da-conocer-categorias-desempeno-2018/

Anderson, M. (2012). The struggle for collective leadership: Thinking and practice in a multi-campus school setting. Educational Management Administration \& Leadership, 40(3), 328-342. https://doi.org/10.1177/1741143212436955 
ARAYA, F. (2015). Rediseño del rol y función del jefe de departamento de asignatura Estudio de caso (Tesis de Magíster). Recuperado de https://repositorio.uc.cl/handle/11534/21526

Barrios, P.; Escare, K.; EspinozA, J. y Jiménez, A. (2018). Liderazgo distribuido un estudio de caso a los beneficios y dificultades de su implementación desde los jefes de departamentos de liceos municipales. (Tesis de licenciatura). Recuperado de http://opac.pucv.cl/pucv_txt/txt-7000/UCC7369_01.pdf

Bernal, A. e IBARRola, S. (2015). Liderazgo del profesor: objetivo básico de la gestión educativa. Revista Ibero-americana de Educação, (67), 55-70. Recuperado en https://rieoei.org/historico/documentos/rie67a03.pdf

BIELECKA, J. (2015). Las características de liderazgo intermedio en el departamento de inglés en un colegio particular privado de alto rendimiento académico. (Tesis de magister). Recuperado de https://repositorio.uc.cl/handle/11534/15788

Bolívar, A. (2019). Una dirección escolar con capacidad de liderazgo pedagógico. Madrid: La Muralla.

CoRTez, M. y Zoro, B. (2016). El rol estratégico de los liderazgos medios al interior de las escuelas para la mejora escolar: Una mirada desde la literatura internacional. Nota Técnica $n .^{\circ} 8$. Chile: Centro de Liderazgo para la Mejora Escolar, Pontificia Universidad Católica de Valparaíso.

CANALES, M. (2006). Metodologías de la investigación social. Santiago: LOM Ediciones.

CANCINo, V. y VerA, L. (2017). Políticas educativas de fortalecimiento del liderazgo directivo: desafíos para Chile en un análisis comparado con países OCDE. Ensaio: Avaliação e Políticas Públicas em Educação, 25(94), 26-58. https://dx.doi.org/10.1590/s0104-40362017000100002

Chávez, S.; Faúndez, M.; Martínez, A. y Zett, I. (2018). El jefe del departamento y las unidades departamentales como promotores del desarrollo profesional docente: un estudio de caso (Tesis de licenciatura). Recuperado de http://opac.pucv.cl/pucv_txt/txt-7000/UCC7407_01.pdf

Cooper, K.; Stanulis, R.; Brondyk, S.; Hamilton, E.; Macaluso, M. \& Meier, J. (2016). The teacher leadership process: Attempting change within embedded systems. Journal of Educational Change, 17, 85-113. https://doi.org/10.1007/s10833-015-9262-4

Crawford, M. (2012). Solo and Distributed Leadership: Definitions and Dilemmas. Educational Management Administration \& Leadership, 40(5), 610-620. https://doi.org/10.1177/1741143212451175

Díaz, C. (2018). Investigación cualitativa y análisis de contenido temático. Orientación intelectual de Revista Universum. Revista General de Información y Documentación, 28(1), 119-142. https://doi.org/10.5209/rgid.60813

FLÜCKIGER, B.; LovetT, S.; DempsteR, N. \& Brown, S. (2015). Middle leaders: Career pathways and professional learning needs. Leading \& Managing, 21(2), 60-74. Recuperado de https://research-repository. griffith.edu.au/handle/10072/341889

Fundación Chile. (2006). Perfil de competencias: Jefe de Departamento. Santiago de Chile: Programa de Educación y Gestión Escolar.

GAIRIN, J. (1999). Los departamentos y equipos educativos como órganos de desarrollo profesional. Profesorado. Revista de currículum y formación del profesorado, 3(1), 1-26. Recuperado de https://redined.educacion.gob.es/xmlui/handle/11162/94941

García, M.; Alvira, F.; Alonso, L. y Escobar, M. (2015). El análisis de la realidad social. Método y técnicas de investigación. Cuarta edición. Madrid: Editorial Alianza.

Grootenboer, P.; Edwards-Groves, C. \& Rönnerman, K. (2015). Leading practice development: Voices from the middle. Professional Development in Education, 41(3), 508-526. https://doi.org/10.1080/19415 257.2014.924985

HaIRON, S.; GoH, J. \& CHUA, C. (2015). Teacher leadership enactment in professional learning community contexts: Towards a better understanding of the phenomenon. School Leadership \& Management, 35(2), 163-182. https://doi.org/10.1080/13632434.2014.992776

HarRis, A.; JAmieson, I. \& Russ, J. (1995). A study of "effective" departments in secondary schools. School Organisation, 15(3), 283-299. https://doi.org/10.1080/0260136950150306 
HarRIs; A.; NG., D.; JonES, M. y THANH, D. (2017). El liderazgo docente en perspectiva: evidencia, implicancias y rumbos futuros. En J. Weinstein y G. Muñoz (Eds.), Mejoramiento y liderazgo en la escuela. Once miradas (227-262). Santiago de Chile: Ediciones Universidad Diego Portales.

Hernández Sampierl, R. y Mendoza, C. (2018). Metodología de la investigación. Las rutas cuantitativa, cualitativa y mixta. Ciudad de México: McGraw-Hill.

Hernández Sampierl, R.; Fernández, C. y Baptista, M. (2010). Metodología de la investigación. Ciudad de México: McGraw-Hill.

Jiménez, V. y Comet, C. (2016). Los estudios de casos como enfoque metodológico. ACADEMO Revista de Investigación en Ciencias Sociales y Humanidades, 3(2). Recuperado de https://dialnet.unirioja.es/ servlet/articulo?codigo $=5757749$

JUNAEB ABIERTA. (s.f.). Indicadores de Vulnerabilidad. Recuperado de http://junaebabierta.junaeb.cl/ catalogo-de-datos/indicadores-de-vulnerabilidad/

JUNAEB. (2018). Prioridades 2018 con IVE SINAE básica, media y comunal. Recuperado de https://www.junaeb.cl/ive

KRICHESKY, G. J. y MURILLO F. J. (2018). La colaboración docente como factor de aprendizaje y promotor de mejora. Un estudio de casos. Educación XX1, 21(1), 135-156. https://doi.org/10.5944/educxx1.20181

Lahtero, T. J.; LÅng, N. \& Alava, J. (2017). Distributed leadership in practice in Finnish schools. School Leadership \& Management, 37(3), 217-233. https://doi.org/10.1080/13632434.2017.1293638

Leitrwood, K. (2016). Department-head leadership for school improvement. Leadership and Policy in Schools, 15(2), 117-140. https://doi.org/10.1080/15700763.2015.1044538

MIFSUD, D. (2017). Distribution dilemmas: Exploring the presence of a tension between democracy and autocracy within a distributed leadership scenario. Educational Management Administration \& Leadership, 45(6), 978-1001. https://doi.org/10.1177/1741143216653974

Montecinos, C.; Aravena, F. y Tagle, R. (2016). Liderazgo Escolar en los Distintos Niveles del Sistema: Notas Técnicas para Orientar sus Acciones. Chile: Centro de Liderazgo para la Mejora Escolar, Pontificia Universidad Católica de Valparaíso.

Sammons, P.; Hillman, J. \& Mortimore, P. (1995). Key characteristics of effective schools: a review of school effectiveness research. Recuperado de https://files.eric.ed.gov/fulltext/ED389826.pdf

Sepúlveda, R. y Volante, P. (2019). Liderazgo Instruccional Intermedio: Enfoques Internacionales para el desarrollo profesional docente en las escuelas chilenas. Profesorado. Revista de Currículum y Formación del Profesorado, 23(3), 341-362. https://doi.org/10.30827/profesorado.v23i3.11231

Supovitz, J. (2018). Teacher leaders' work with peers in a Quasi-formal teacher leadership model. School Leadership \& Management, 38(1), 53-79. https://doi.org/10.1080/13632434.2017.1389718

Tichnor-Wagner, A.; Harrison, C. \& Cohen-Vogel, L. (2016). Cultures of learning in effective high schools. Educational Administration Quarterly, 52(4), 602-642. https://doi.org/10.1177/0013161X16644957

Valenzuela, J. P.; Bellel, C. \& Allende, C. (2016). Measuring systematic long-term trajectories of school effectiveness improvement. School Effectiveness and School Improvement, 27(4), 473-491. https://doi.org/10.1080/09243453.2016.1150861

Vanblaere, B. \& Devos, G. (2016). Relating school leadership to perceived professional learning community characteristics: A multilevel analysis. Teaching and Teacher Education, 57, 26-38. https://doi.org/10.1016/j.tate.2016.03.003

WeNnER, J. \& CAMPBeLL, T. (2017). The theoretical and empirical basis of teacher leadership: A review of the literature. Review of Educational Research, 87(1), 134-171. https://doi.org/10.3102/0034654316653478

York-BARR, J. \& Duke, K. (2004). What Do We Know about Teacher Leadership? Findings from Two Decades of Scholarship. Review of Educational Research, 74(3), 255-316.

https://doi.org/10.3102/00346543074003255 